This manuscript is contextually identical with the following published paper

Saak V. Ovsepian, Károly Elekes, From network architecture of forebrain systems to brain wide web, Basal Ganglia, Volume 5, Issue 1, March 2015, Pages 11-14, ISSN 2210-5336, http://dx.doi.org/10.1016/j.baga.2015.02.001.

The original published pdf available in this website:

http://www.sciencedirect.com/science/article/pii/S2210533615000039

\title{
Title: From Network Architecture of Forebrain Systems to Brain
}

\section{Wide Web}

\section{Authors: Saak V. Ovsepian ${ }^{1,2}$ and Károly Elekes ${ }^{3}$}

Addresses: Department of Translational Brain Research, German Centre for Neurodegenerative

Diseases (DZNE) Munich, Germany (1); Faculty of Science and Health, School of Biotechnology and International Centre for Neurotherapeutics, Dublin City University, Dublin, Republic of Ireland (2) and Department of Experimental Zoology Balaton Limnological Institute, MTA Centre for Ecological Research, Hungarian Academy of Sciences, Tihany, Hungary (3)

Corresponding authors: Saak Ovsepian: saak.ovsepian@gmail.com and Karoly Elekes: elekes@,okologia.mta.hu 


\begin{abstract}
A conference was organized by the Hungarian Academy of Sciences in honour of Professor Laszlo Zaborszy, to recapitulate the progress and identify principal directions for future studies of circuit architecture and connections in forebrain systems. Neuroscientists gathered in Tihany to present and debate their latest discoveries of the organizational rules and functions of local and long-range basal forebrain projections, with their appraisal in the context of network theory. Here, we report on the main themes in basic and translational research that were discussed and reflect on conceptual and technical developments in untangling the inner design and mechanisms of deep forebrain circuits.
\end{abstract}

\title{
Key words
}

Forebrain systems, neuronal circuits, network architecture, neuro-informatics, basalo-cortical, prefrontal cortex 


\section{Seeking order in the 'jumble' of basal forebrain neurons}

In his brief histological account of the anterior perforate substance, Santiago Ramon y Cajal compared the bulky and abundantly Nissl reactive nerve cells to motor neurons. What he did not realize was that these neurons belong to the same system as the large and loosely scattered cells of the diagonal band of Broca (Ramón y Cajal, 1911). However, Ramón y Cajal understood that the large neurons of the septal nuclei were associated with the hippocampus. It was Brockhaus (1942), who for the first time explicitly recognized that the large neurons dispersed from the rostral pole of the medial septum to the sub-thalamic area actually belong to the same anatomical organization, known as the basal forebrain (Zaborszky, 2002). The gradual emergence of the remarkable diversity of neuronal types and synaptic connections within the basal forebrain systems required in-depth research and detailed mapping. This was one of the key scientific objectives of Prof. Zaborszky from the University of New Jersey, Rutgers (USA). Discovering the out of sight order within neuronal assemblies and their connectivity has been in the research spotlight of his laboratory for over many decades. In a series of papers, Zaborszky demonstrated an exquisite organization in the outwardly muddled construction of the basal forebrain circuits. He put forward strong and systematic evidence, which demonstrates that similar to basal ganglia circuits, there is stringent segregation of neuronal types and circuits in this brain region, capable of parallel processing and allocation of information in a self-regulatory fashion (Zaborszky et al., 2013). More recent collaborative work with Prof. Zilles of Julich Research Centre (Germany) led to the development of a method, that allows accurate extrapolation of the volume of the nucleus basalis Meynert of the basal forebrain from MRI scans (Zaborszky et al., 2008). This discovery makes feasible the monitoring of functional 
dynamics within this furtive forebrain region, known to be severely compromised in Alzheimer's disease.

The detailed account of the intrinsic organization and connectivity of the basal forebrain was complemented by in-depth analysis of their evolutionary and ontogenetic characteristics by Prof. Medina from the University of Lleida (Spain). The main tenets of Medina's research focuses on (1) the phylogenic conservation of the forebrain modulator systems (including cholinergic and catecholaminergic) in vertebrates and (2) strict segregation of neuronal groups within the basal ganglia and amygdalar structures, with identification of distinctive sites of their ontogenetic origin and discrete migrational paths, already defined during the early phylogeny (Medina et al., 2014). The unparalleled advances made recently in exploring the architecture and connectivity of forebrain systems, without a doubt, reflect recent developments in imaging tools and recombinant technologies that enabled tracing and visualization of individual neurons with their development and plasticity. This was reflected in vivid slides shown by Prof. Wouterlood from Vrije University (Netherlands), who reviewed the principal methods for labelling and real time imaging of stationary and dynamic neurons and their fine elements at light and electron microscopic levels. The main emphasis was placed on fourth generation tracing techniques and use of the recombinant technologies with transgenic models (Wouterlood et al., 2014). Prof. Wouterlood stressed that the technological advances in these directions in the near future should enable further in-depth analysis of structural and functional traits of selective sub-populations of neurons, including those of the basal ganglia and forebrain, with manipulation of their activity, using optogenetic tools.

\section{Deciphering the organizational principles of forebrain circuits}

The notion of parallel organization of cortico-basal circuits was explicitly formulated over two 
decades ago by Alexander and Crutcher (Alexander and Crutcher, 1990). Detailed analysis of the forebrain network architecture and connectivity has been in the focus of research lead by Prof. Haber at the University of Rochester (USA). In contrast to the established view of cortico-basal circuits as parallel processors of independent streams of signals (associative, motivational and motor), Prof. Haber's work revealed incredible organizational complexity and interconnections within these networks. In addition to semi-autonomous allocation and processing of information, profuse local connections ensure tight functional integration between parallel circuits. These previously unrecognized details of basal ganglia circuits, explained Prof. Haber, emerge to be of prime importance for learning and adaptive behaviour as well as for the optimization of goal driven activities, with evaluation of reward signals. The in-depth characterization of circuits that integrate executive cortical programs with basal ganglia are likely to facilitate the understanding of their operational principles and possibly lead to the development of tools for manipulating their functions (Haber and Behrens, 2014 ). Prof. Haber stressed the importance of comparing functional imaging data obtained in human studies with primate tracing information on corticobasal connectivity, to avoid misinterpretation of imaging data.

Detailed analysis of the architecture and organization of cortical and sub-cortical structures along with their synaptic connectivity has also been the main subject of research directed by Prof. Barbas at Harvard Medical School (USA). The prefrontal cortex with associated networks remained the focus of her work over recent years, with experiments conducted in accordance with a theoretical framework of developing structural models, which allow prediction of the pattern of neural connections. The prime objective of current research is discovery of organizational rules and mechanisms of prefrontal circuits associated with memory, cognition and emotional behaviour in primates, to identify fine synaptic and network alterations related with an array of neuropsychiatric disease, including autism and schizophrenia 
(Zikopoulos and Barbas, 2013).

No doubt, Prof. Napier of Rush University (Chicago, USA) was cognizant how changes in forebrain circuits can be related to addictive behaviour and compulsive drug use. Her research extends from molecular neurobiology and biochemistry to neurophysiology and behavioural studies, using animal models of human disease. Limbic system with related networks was the prime theme of her presentation, with main emphasize placed on the complex interplay between the glutamatergic, GABAergic and dopaminergic circuits. Recent evidence from her group suggests that a deficit in multi-part regulation of the activity within the prefrontal cortex accumbens - hippocampal axis contributed by functional aberrations in pallidial GABAergic and tegmental dopaminergic systems promotes abnormal reward-motivated behaviours (Napier and Grace, 2014). Prefrontal cortex and basal ganglia circuits have also been in the limelight of research led by Prof. Groenewegen at the Vrije University (Netherlands). His untiring efforts led to unravelling of organizational principles and functional mechanisms of deep brain networks involved in cognitive, emotional and motivational behaviours. His recent work put an innovative 'spin' on the old core-shell dichotomy of the nucleus accumbens proposed several decades ago by Zaborszky and co-workers (Zaborszky et al., 1985; Voorn et al., 2004). In his studies, Prof. Groenewegen tackled some of the most unsettled issues of forebrain circuits and functions, using animal models and imaging of the human brain, with the main objective to find out structural and functional alterations in deep brain circuits, with the aim of developing effective treatments for an array of neurological and psychiatric disorders involving deep forebrain structures (van den Heuvel et al., 2010). The theme of the research presented by Prof. Witter of Institute of Science and Technology (Norway) was the topographical organization and timing of the development of forebrain circuits and connections in the course of early ontogeny, using the entorhinohippocampal model of rodents. The earlier collaborative research of Prof. Witter into the variety 
of neuron types in these brain structures with their role in learning and spatial navigation contributed to the Nobel Prize Winning discovery of grid cells in the entorhinal cortex (Sargolini et al., 2006). From the most recent studies directed by Prof. Witter, it emerged that the topographical organization and connections of entorhinal-hippocampal circuits in rats stabilize very early during their development and are fully established already at the first week of postnatal life (O'Reilly et al., 2013). The specificity and stability of projections within this brain region, explained Prof. Witter, strongly suggests that both the architecture and connections that form during early development will be maintained throughout adulthood.

\section{Towards translational research of forebrain circuits}

Moving to translational studies of forebrain systems, Prof. Morales from National Institute of Drug Abuse at Baltimore (USA) presented data on the molecular and functional alterations occurring in neurons and synapses associated with habit formation and addictive behaviour. Neuronal circuits with connections in the ventral tegmental area (VTA) have been the focus of recent research in Morales laboratory, through the use of neuro-anatomical, molecular biological and electrophysiological tools. In addition to well established GABAergic and dopaminergic cells, most recent experimentations have revealed a large amount of glutamatergic neurons in this key dopaminergic structure (Yamaguchi et al., 2013), with their functionality remaining elusive. Interestingly, rising evidence implies a close relation between forebrain circuits involved in stress with those implicated in drug abuse, with VTA nuclei holding a strategic position.

Prof. Cullinan of Marquette University (USA) gave a complementary talk on translational aspects of forebrain circuits with their involvement in stress-related disorders. Based on recent discoveries, following ideas were proposed: (1) corticotropine-releasing neurons $(\mathrm{CRH})$ in the para-ventricular hypothalamic nucleus are closely integrated within forebrain circuits and (2) 
CRH neurons that mediate stress response receive strong feedback inhibition from local GABAergic networks, which demonstrate a remarkable plasticity and are enlarged in animal models under chronic exposure to stressors. Prof. Cullinan clarified that while discovery of the details of hypothalamic and associated circuitry facilitated the understanding of the relation between the neuronal mechanisms of stress and several neuropsychiatric disease, the plasticity of GABAergic circuits provides a new therapeutic window for intervention with stress response, using selective modulators of the activity of local GABAergic network (Myers et al., 2014).

The translational aspects of forebrain dopaminergic circuits were also the focus of studies led by Prof. Lanciego from the University of Navara, Pomplona (Spain). Through large scale screening of G-protein coupled receptors in the basal ganglia and forebrain in non-human primates along with analysis of structural details and synaptic morphology in normal and Parkinson's disease models, Prof. Lanciego aim to identify early and specific alterations in basal ganglia neurons and circuits, which predict the commencement of clinical phenotype. This knowledge should assist in understanding the pathobiology of Parkinson's disease and related disorders with their early diagnosis to facilitate the development of counteracting therapeutics (Sierra et al., 2014), including viral based treatments.

\section{Single synapse - neuronal networks - functional imaging of the brain}

Synapse constitutes the prime site of neuronal signalling and integration. The ultra-structural details and functional characteristics of the glutamatergic synapse were in the spotlight of two presentations. Prof. Tamás from University of Szeged (Hungary) reported that over years his group gathered and is in the process of analysing of data from an impressive $\sim 14000$ pairs and triplets of connected neurons. This unique library of data provides an unprecedented source of information about the operational principles of central synapses. New evidence was also 
presented in support of recently identified neuro-gliaform cells. In depth characterization of these natural 'hybrids' suggests that they play critical role in stabilizing the activity of local cortical networks, via diffuse release of GABA and paracrine effects. Also, data has been obtained, which suggests these chimeric cells as a major source of cortical insulin (Molnar et al., 2014). The structure and functions of glutamatergic synapses constituted also one of the main research objectives of studies led by Prof. Nusser from Institute of Experimental Medicine, Hungarian Academy of Sciences (Hungary). He presented new quantitative, electron microscopic and immune-gold localization data, which allowed precise assessment of the number and location of receptors and ion channels at individual central synapses. In-depth studies of the functional and structural features of cortical glutamatergic synapses by Prof. Nusser and his team have led to the discovery of a strong correlation between the size of the presynaptic active zone and the strength of the individual synaptic connection (Holderith et al., 2012).

One of the most influential concepts of central neural mechanisms is regulation of cortical activity and processing of external inputs by ascending non-specific synaptic drive from the reticular core of the brain, as proposed by Moruzzi and Magoun (Moruzzi and Magoun, 1949). Decades of meticulous research from many laboratories, including landmark discoveries made by Prof. Detári from Eötvös Loránd University (Hungary) revealed the highly complex relationship between the single unit activity in the basal forebrain and large scale dynamics of cortical network. Use of more sophisticated tools and methods, projected Prof. Detári, should lead to the discovery of further diversities amongst the reach varieties of basal forebrain neurons and reveal important aspects of their functional relationship with complex dynamics of cortical circuits (Detári, 2000). His collaboration with Prof. Zaborszky's group led to the discovery of the significant correlation of the parameters of cortical EEG with activity of subgroups of neurons in the basal forebrain (Duque et al., 2000). Data presented by Prof. Semba from Halifax, Nova 
Scotia University (Canada), another pioneer in studies of basal forebrain circuits, focused on the mechanisms and functions of forebrain circuits involved in the regulation of circadian rhythms, sleep-wake cycles and related neuronal dynamics. Prof. Semba emphasized the close relationship between the circadian and sleep-wake brain systems and provided new data, which demonstrate how chronic impairments of these basic biological processes can influence the health and behaviour of experimental animals (Deurveilher et al., 2013). Recent work from her group imply critical role of astrocytes in regulation of the sleep and circadian activity.

As a key extra-thalamic relay, cholinergic basal forebrain nuclei attracted considerable interest also as a fast and powerful modulator of cortical response to external signals, a process implicated in cognitive task shifting and attention. Prof. Nunez from the University of Autonoma Madrid (Spain) has made a strong contribution towards the discovery of circuits and mechanisms underlying the ascending modulator effects of basalo-cortical projections on cortical mechanisms. Combining in vivo recordings of electrical activity with forebrain stimulation, he with his team has taken recently a lead in identifying the effects of specific networks and demonstrated key role of inputs from preforontal cortex to the basal forebrain in modulating cortical processing of thalamic signals. He uses optogenetic stimulation of basal forebrain neurons to dissect the role of various neuronal groups and circuits in shaping the activity of cortical networks (Nunez et al., 2012). Whether subtle changes in large scale activity of cortical and subcortical networks can predict slowly progressing cognitive decline followed by breakdown of integrative brain functions in Alzheimer's disease and related dementias has been the subject of research directed by Prof. Cantero at Pablo de Olavide University (Spain). His recent studies aimed on defining alterations in the activity patterns and functions associated with aging and neurodegenerative disease, using functional brain imaging in humans. In depth analysis of slight changes in the activity of neural networks associated with metabolic decline 
and accumulation of $A \beta$ prior to the onset of degenerative changes, explained Prof. Cantero, should provide clinicians with first-rate opportunities to identify those at risk and proceed with the counteracting therapies, to slow down the progression of the cognitive decline and counteract the advancement of irreversible degenerative changes in the course of Alzheimer's disease (Sanchez-Espinosa et al., 2014).

\section{Complex network theory in service for neuroscience research: Brain Wide Web}

Understanding the organization and operational principles of complex networks should enable manipulations and ultimately control of their behaviour, explained Prof. Albert-László Barabási from Harvard and Centre for Complex Network Research at Northeastern Universities (USA). His studies have been widely recognized amongst network theory specialists, while the breakthrough discovery of scale-free networks provided a powerful model (acknowledged as Barabási-Albert model) for exploring the cause for constant emergence of new networks in natural, technological and social systems, including those such as Internet, Facebook and others. Developing vigorous mathematical tools to model and control complex network dynamics has been in the spotlight of Prof. Barabási recent research. The research towards finding means for influencing networks, according to Barabási, should lie through merger of matching theory with dynamical control problems. In such a way, one may obtain insights as to how identification of the points of manipulation can assist in recapitulating the architecture of the complex system at a given time point and predicting its evolution and behaviour (Newman et al., 2006).

Taking advantage of the operational principles of networks and their application for management and free exchange of experimental data has been the subject of studies of fervent proponent of neuro-informatics, Prof. Bjaalie from the University of Oslo (Norway). His work focused on identifying the principles and patterns of neuronal wiring, which underlie formation 
and transformation of cortical representations as well as development of computational tools for their multi-level mapping. Prof. Bjaalie reflected on the Rodent Brain Navigator, a database he and his collaborators introduced recently to facilitate the storing, retrieval and analysis of multiple categories of Neuroscience data. This Oracle-based portal runs on an Apache Tomcat server, and in addition to high-resolution histochemical imaging maps intends to incorporate the volumetric measurements collected with MRI or PET scans, along with molecular and functional data. The database will also provide metadata describing animals and experimental methods and will be constantly updated by a consortium of laboratories (Bjaalie et al., 2008). The quality of the material will be vigorously controlled by peer review, to ensure that the most up to date and accurate information is available to the scientific community and public.

Acknowledgements: Authors thank Dr. Valerie B. O'Leary for careful proofreading of this manuscript. Publication costs were covered by NIH/NINDS NS023945 grant to Prof. Zaborszky.

\section{References}

Alexander GE, Crutcher MD (1990) Functional architecture of basal ganglia circuits: neural substrates of parallel processing. Trends Neurosci 13:266-271.

Bjaalie JG, Grillner S, Usui S (2008) Neuroinformatics: databases, tools, and computational modeling for studying the nervous system. Neural Netw 21:1045-1046.

Brockhaus H (1942) Vergleichend-anatomische Utersuchungen uber den Basalkern Komplex. . J Psychol Neurol Leipzig 51:57-95.

Detari L (2000) Tonic and phasic influence of basal forebrain unit activity on the cortical EEG. Behav Brain Res 115:159-170. 
Deurveilher S, Ryan N, Burns J, Semba K (2013) Social and environmental contexts modulate sleep deprivation-induced c-Fos activation in rats. Behav Brain Res 256:238-249.

Duque A, Balatoni B, Detari L, Zaborszky L (2000) EEG correlation of the discharge properties of identified neurons in the basal forebrain. J Neurophysiol 84:1627-1635.

Haber SN, Behrens TE (2014 ) The Neural Network Underlying Incentive-Based Learning: Implications for Interpreting Circuit Disruptions in Psychiatric Disorders. Neuron 3:1019-1039.

Holderith N, Lorincz A, Katona G, Rozsa B, Kulik A, Watanabe M, Nusser Z (2012) Release probability of hippocampal glutamatergic terminals scales with the size of the active zone. Nat Neurosci 15:988-997.

Medina L, Abellán A, Vicario A, Desfilis E (2014) Evolutionary and developmental contributions for understanding the organization of the basal ganglia. Brain Behav Evol $83: 112-125$.

Molnar G, Farago N, Kocsis AK, Rozsa M, Lovas S, Boldog E, Baldi R, Csajbok E, Gardi J, Puskas LG, Tamas G (2014) GABAergic neurogliaform cells represent local sources of insulin in the cerebral cortex. J Neurosci 34:1133-1137.

Moruzzi G, Magoun HW (1949) Brain stem reticulata formation and activation of the EEG. Electroencephalogr Clin Neurophysiol I :455-473.

Myers B, Mark Dolgas C, Kasckow J, Cullinan WE, Herman JP (2014) Central stress-integrative circuits: forebrain glutamatergic and GABAergic projections to the dorsomedial hypothalamus, medial preoptic area, and bed nucleus of the stria terminalis. Brain Struct Funct 219:1287-1303.

Napier T, Grace AA (2014) Linking neuroscience with modern concepts of impulse control disorders in Parkinson's disease. . Mov Disorders In press. 
Newman M, Barabási AL, Watts DJ (2006) The Structure and Dynamics of Networks. New York: Princeton University Press

Nunez A, Dominguez S, Buno W, Fernandez de Sevilla D (2012) Cholinergic-mediated response enhancement in barrel cortex layer V pyramidal neurons. J Neurophysiol 108:1656-1668.

O'Reilly KC, Gulden Dahl A, Ulsaker Kruge I, Witter MP (2013) Subicular-parahippocampal projections revisited: development of a complex topography in the rat. J Comp Neurol 521:4284-4299.

Ramón y Cajal S (1911) Histologie du système nerveux de l'hommeet des vertébrés. Paris, France.

Sanchez-Espinosa MP, Atienza M, Cantero JL (2014) Sleep deficits in mild cognitive impairment are related to increased levels of plasma amyloid-beta and cortical thinning. Neuroimage 98:395-404.

Sargolini F, Fyhn M, Hafting T, McNaughton BL, Witter MP, Moser MB, Moser EI (2006) Conjunctive representation of position, direction, and velocity in entorhinal cortex. Science 312:758-762.

Sierra S, Luquin N, Rico AJ, Gomez-Bautista V, Roda E, Dopeso-Reyes IG, Vazquez A, Martinez-Pinilla E, Labandeira-Garcia JL, Franco R, Lanciego JL (2014) Detection of cannabinoid receptors $\mathrm{CB} 1$ and $\mathrm{CB} 2$ within basal ganglia output neurons in macaques: changes following experimental parkinsonism. Brain Struct Funct DOI 10.1007/s00429014-0823-8.

van den Heuvel OA, van der Werf YD, Verhoef KM, de Wit S, Berendse HW, Wolters E, Veltman DJ, Groenewegen HJ (2010) Frontal-striatal abnormalities underlying behaviours in the compulsive-impulsive spectrum. J Neurol Sci 289:55-59. 
Voorn P, Vanderschuren LJ, Groenewegen HJ, Robbins TW, Pennartz CM (2004) Putting a spin on the dorsal-ventral divide of the striatum. Trends Neurosci 27:468-474.

Wouterlood FG, Bloem B, Mansvelder HD, Luchicchi A, Deisseroth K (2014) A fourth generation of neuroanatomical tracing techniques: exploiting the offspring of genetic engineering. J Neurosci Methods 235:331-348.

Yamaguchi T, Wang HL, Morales M (2013) Glutamate neurons in the substantia nigra compacta and retrorubral field. Eur J Neurosci 38:3602-3610.

Zaborszky L (2002) The modular organization of brain systems. Basal forebrain: the last frontier. Prog Brain Res 136:359-372.

Zaborszky L, Alheid GF, Beinfeld MC, Eiden LE, Heimer L, Palkovits M (1985) Cholecystokinin innervation of the ventral striatum: a morphological and radioimmunological study. Neuroscience 14:427-453.

Zaborszky L, Hoemke L, Mohlberg H, Schleicher A, Amunts K, Zilles K (2008) Stereotaxic probabilistic maps of the magnocellular cell groups in human basal forebrain. Neuroimage 42:1127-1141.

Zaborszky L, Csordas A, Mosca K, Kim J, Gielow MR, Vadasz C, Nadasdy Z (2013) Neurons in the Basal Forebrain Project to the Cortex in a Complex Topographic Organization that Reflects Corticocortical Connectivity Patterns: An Experimental Study Based on Retrograde Tracing and 3D Reconstruction. Cereb Cortex.

Zikopoulos B, Barbas H (2013) Altered neural connectivity in excitatory and inhibitory cortical circuits in autism. Front Hum Neurosci 27:609. 